\title{
Efeito da moagem de alta energia na morfologia e compressibilidade do compósito $\mathrm{Mo}-30 \% \mathrm{Cu}$
}

\section{Effect of high energy milling on the morphology and compressibility of Mo- $30 \%$ Cu composite}

\author{
Camila dos Santos Torres \\ Eng. a Metalúrgica, Laboratório de Transformação Mecânica, Departamento de Metalurgia, UFRGS \\ Av. Bento Gonçalves, 9500 \\ Porto Alegre, RS, Brasil CEP 91501-970 \\ camila.torres@ufrgs.br
}

Fábio Luis Knewitz

Msc. Eng. Metalúrgico, doutorando. Laboratório de Transformação Mecânica, Departamento de Metalurgia, UFRGS

Av. Bento Gonçalves, 9500

Porto Alegre, RS, Brasil, CEP 91501-970

fábio.knewitz@ufrgs.br

Wilberth Harold Deza Luna

Msc. Eng. Metalúrgico, doutorando. Laboratório de Transformação Mecânica, Departamento de Metalurgia, UFRGS

Av. Bento Gonçalves, 9500

Porto Alegre, RS, Brasil, CEP 91501-970

harold.luna@ufrgs.br

Alexandre da Silva Rocha

Prof. Dr. Eng. Laboratório de Transformação Mecânica, Departamento de Metalurgia, UFRGS

Av. Bento Gonçalves, 9500

Porto Alegre, RS, Brasil, CEP 91501-970

alexandre.rocha@ufrgs.br

Lirio Schaeffer

Prof. Dr. Eng. Laboratório de Transformação Mecânica, Departamento de Metalurgia, UFRGS

Av. Bento Gonçalves, 9500

Porto Alegre, RS, Brasil, CEP 91501-970

schaefer@ufrgs.br

\section{Resumo}

Este trabalho tem por objetivo a investigação do efeito da moagem de alta energia sobre a morfologia, tamanho de partícula e compressibilidade do compósito Mo-Cu. O compósito Mo-Cu combina a alta resistência mecânica do Mo com a alta condutividade térmica e elétrica do $\mathrm{Cu}$. Estas características são importantes para dissipadores de calor e condutores elétricos. A moagem de alta energia produz pós compósitos com alto nível de homogeneização e refina o tamanho de

\section{Abstract}

The purpose of this work is to analyze the effects of high energy milling on the granulometry, powder morphology and compressibility of $\mathrm{Mo}-\mathrm{Cu}$ composite. The Mo-Cu composite combines high mechanical strength of Mo with the high thermal and electrical conductivity of $\mathrm{Cu}$. These characteristics are important to heat sink and electrical conductors. The high energy milling produces composite powders with high homogeneity and refines the grain size. The synthesis of Mo-30Cu composite, starting from powders of $\mathrm{Mo}$ and $\mathrm{Cu}$, was realized for milling times of 
grão. A síntese do compósito Mo-30Cu, partindo dos pós de $\mathrm{Mo}$ e $\mathrm{Cu}$, foi realizada para tempos de moagem de $1,4,8,16$ e 32 horas. A fim de observar a influência do tempo de moagem sobre a microestrutura, empregaram-se as técnicas de microscopia eletrônica de varredura e granulometria a laser. Esta avaliação permitiu observar o comportamento morfológico e a interação entre os elementos do material produzido. Os resultados mostram que com o decorrer do tempo de moagem ocorreu a diminuição do tamanho de partículas, que demonstra a viabilidade de obtenção deste compósito.

Palavras-chave: metalurgia do pó, compósito Mo$\mathrm{Cu}$, moagem de alta energia.
$1,4,8,16$ and 32 hours. Scanning electron microscopy and laser granulometry were used to follow the influence of milling time on the microstructure. This allowed to observe the morphological evaluation and interaction between the elements of the material produced. The results show that the particle size and compressibility decreased with the milling time, which shows the viability to obtain this composite by the proposed process.

Key words: conventional powder metallurgy, Mo-Cu composite, high energy milling.

\section{Introdução}

Atualmente, novos materiais que possuam propriedades como alta tenacidade, alta dureza e boa usinabilidade, têm recebido a atenção de pesquisadores, e métodos de fabricação mais eficazes têm sido aplicados com objetivo de melhorar as propriedades desses novos materiais (Cai et al., 2007).

Em materiais utilizados como contatos elétricos, procura-se combinar as características de alta condutividade elétrica de metais como o cobre e a prata, com as características de alta resistência ao calor, elevada resistência ao desgaste e elevada resistência ao efeito da formação de arco dos metais refratários, tais como, tungstênio e o molibdênio (Rotta, 2005).

O cobre é um dos materiais mais importantes para aplicações térmicas e elétricas. A sua utilização vai desde cabos e transformadores de linha de transmissão e instalações prediais a aparelhos elétricos e eletrônicos e seus componentes. Tem maior condutividade térmica e elétrica e um menor coeficiente de expansão térmica (CTE) do que o alumínio (Schubert et al., 2008).

O molibdênio é um metal refratário de interesse para aplicações que envolvam altas temperaturas, por possuir alto ponto de fusão $\left(2623^{\circ} \mathrm{C}\right)$, no entanto, isto torna extremamente difícil seu processamento por fundição (Song et al., 2008). Devido à baixa sinterabilidade de sistemas com metais refratários, a utilização de pós ultrafinos e homogêneos podem melhorar a sinterabilidade deste material, especialmente na sinterização em fase líquida de sistemas como o Mo-Cu em que o mecanismo dominante na sinterização é o rearranjo das partículas (Martinez et al., 2007).

Nos últimos anos, a mistura mecânica e a moagem de alta energia têm sido utilizadas para preparar materiais compósitos, a fim de obter microestruturas homogêneas. As propriedades elétricas dos materiais de contato, como alta condutividade térmica para dissipar o calor gerado pelo arco e pela passagem de corrente elétrica, estabilidade química para evitar a formação de camadas isolantes de óxidos, sulfetos e outros, e alta resistência ao arco voltaico, são fortemente influenciadas pela microestrutura do 
material. O refinamento da microestrutura é importante para melhorar o desempenho de materiais de contato, incluindo a resistência mecânica e condutividade elétrica (Chen et al., 2005).

O presente trabalho faz um estudo sobre o compósito Mo-30\%Cu preparado por moagem de alta energia em um moinho do tipo atritor. Durante a moagem, amostras de pós foram coletadas após 1, 4, 8, 16 e 32 horas para investigar a evolução morfológica das partículas durante a síntese e o efeito da variação do tempo de moagem sobre o tamanho de partícula e compressibilidade das misturas. Para caracterização dos pós produzidos foram realizadas análises por microscopia eletrônica de varredura (MEV) e granulometria a laser.

\section{Materiais e métodos}

Os pós elementares usados no presente trabalho foram molibdênio e cobre. Na Tabela 1 estão listadas as características do pó de partida. Os pós na proporção de $70 \%$ Mo e $30 \%$ Cu foram misturados em um moinho do tipo atritor com o objetivo de obter uma mistura homogênea e alta formação de partículas compósitas.

Tabela 1: Características dos pós de partida.

\begin{tabular}{|c|c|c|c|}
\hline Pó & Tamanho de Partícula & $\begin{array}{c}\text { Pureza } \\
\text { (\% atômica) }\end{array}$ & Formato \\
\hline $\mathrm{Mo}$ & $<0,037 \mathrm{~mm}$ & $85,5 \%$ & Arredondada \\
\hline $\mathrm{Cu}$ & $<0,044 \mathrm{~mm}$ & $99,5 \%$ & Dendrítico \\
\hline
\end{tabular}

Os pós foram moídos via úmida em hexano, utilizando esferas de aço e velocidade rotacional de 800 rpm sob atmosfera de hidrogênio, sendo o moinho atritor utilizado da marca NETZSCH modelo MOLINEX PE 5. Amostras de pós foram coletadas após diferentes tempos de moagem: 1, 4, 8, 16 e 32 horas.

As amostras foram caracterizadas quanto a sua morfologia por microscopia eletrônica de varredura (MEV) e tamanho de partícula por granulômetro a laser. A distribuição granulométrica foi determinada por espalhamento de luz, através da utilização do granulômetro a laser Cilas 1064. As medidas são realizadas em soluções líquidas contendo o material particulado em suspensão, sendo a contagem efetuada via microcomputador acoplado com interface serial.

Para a determinação da compressibilidade foram confeccionados corpos de prova em matriz cilíndrica sendo utilizada uma máquina de ensaios universal marca Kratos com capacidade máxima de 10 tonf. A aquisição dos dados adquiridos pelos sensores (célula de carga e sensor de deslocamento tipo Ivdt) foi realizada através do sistema Spider 8, da HBM, com o uso do programa Catman Express. Todos os ensaios foram realizados a temperatura ambiente. 
Efeito da moagem de alta energia na morfologia e compressibilidade do compósito Mo-30\%Cu

\section{Resultados e discussão}

\subsection{Caracterização dos pós de partida}

A Figura 1 mostra a micrografia do pó de molibdênio obtida por microscopia eletrônica de varredura, exibindo a forma típica, a distribuição de tamanho de partícula e o estado de aglomeração do material. Observa-se para o molibdênio uma morfologia arredondada e tamanhos de partículas bastante heterogêneos.
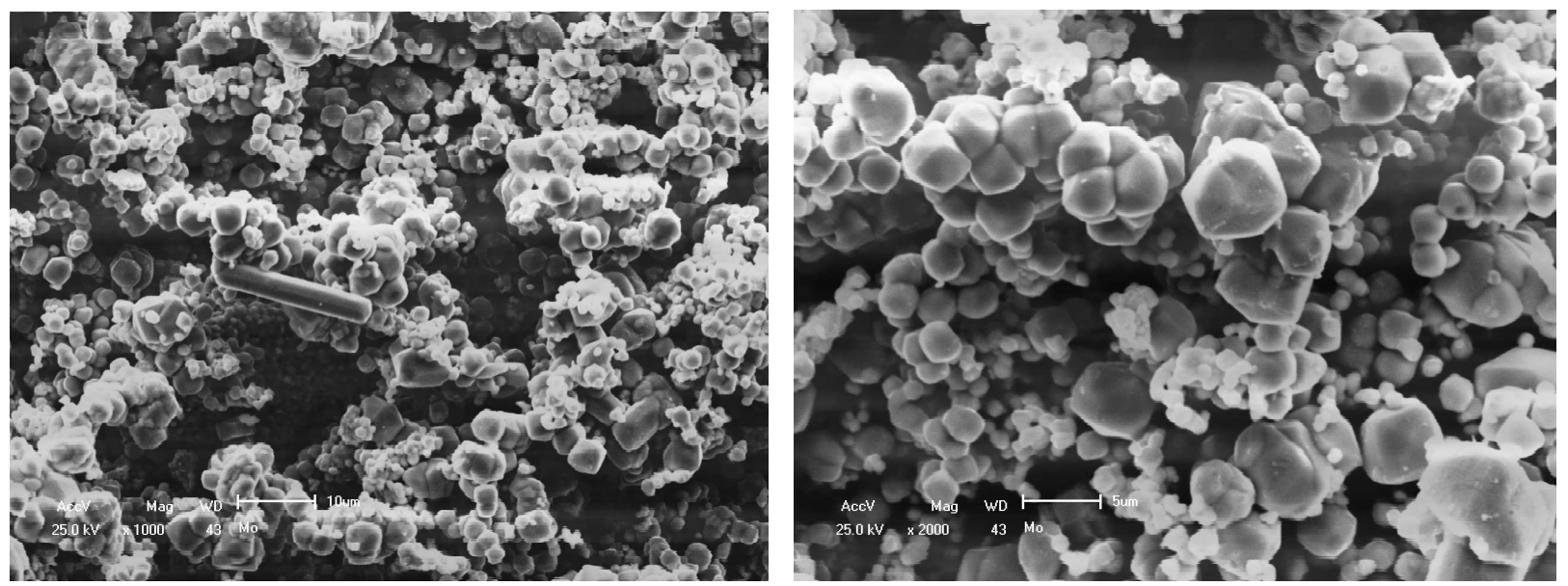

Figura 1: Micrografia do pó de molibdênio. Aumento de 1000x e 2000x respectivamente.

Na Figura 2 são apresentadas as micrografias do pó de cobre. Nota-se para o cobre, que as partículas possuem morfologia dendrítica com tamanhos de partículas bastante homogêneos.
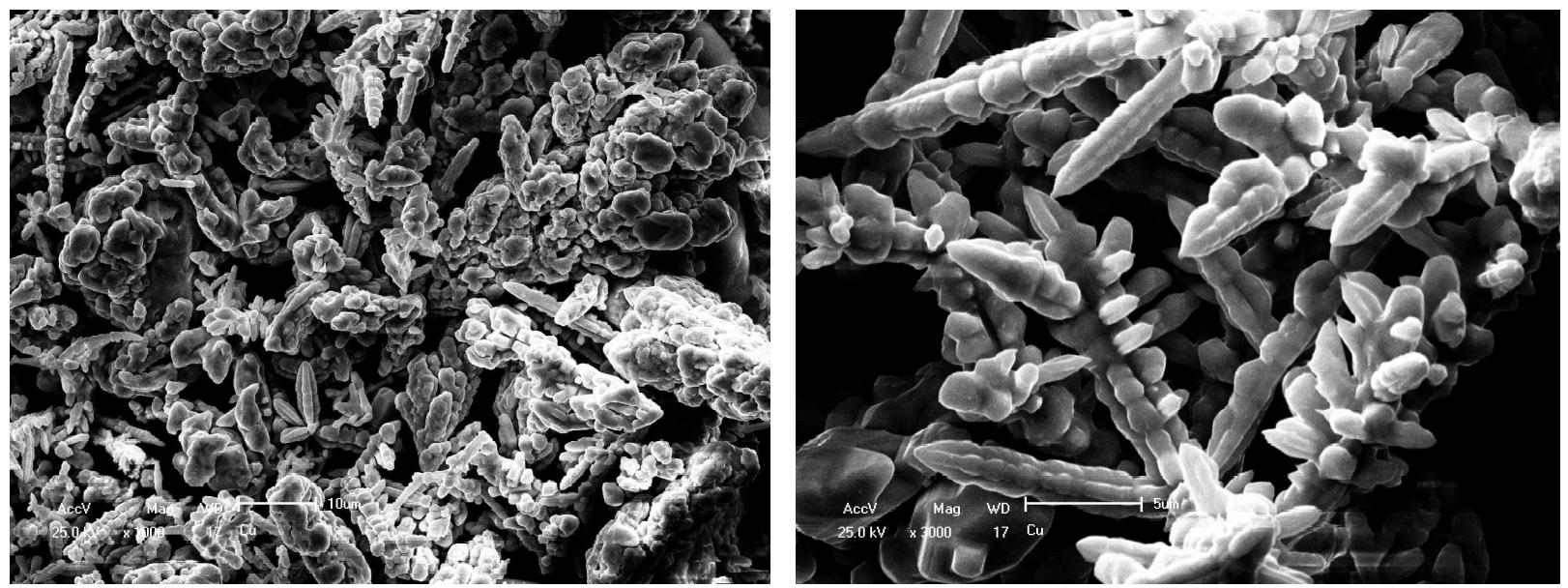

Figura 2: Micrografia dos pó de cobre. Aumento de 1000x e 3000x respectivamente 


\subsection{Efeito do tempo de moagem no tamanho de partícula}

Tamanhos característicos às proporções em que 10, 50 e $90 \%$ do material é passante, foram determinados e representados respectivamente por $D_{10}, D_{50}$ e $D_{90}$. Os resultados estão apresentados na Tabela 2.

Tabela 2: Tamanho médio de partículas.

\begin{tabular}{|c|c|c|c|}
\hline \multirow{2}{*}{$\begin{array}{c}\text { Tempo de } \\
\text { MAE }\end{array}$} & \multicolumn{3}{|c|}{ Tamanho Médio de Partículas $(\boldsymbol{\mu m})$} \\
\cline { 2 - 4 } & $\mathbf{D}_{\mathbf{1 0}}$ & $\mathbf{D}_{\mathbf{5 0}}$ & $\mathbf{D}_{\mathbf{9 0}}$ \\
\hline 1 hora & 5,29 & 14,23 & 41,47 \\
\hline 4 horas & 5,32 & 16,04 & 36,74 \\
\hline 8 horas & 4,55 & 13,58 & 32,09 \\
\hline 16 horas & 4,38 & 11,72 & 26,25 \\
\hline 32 horas & 1,82 & 4,81 & 10,79 \\
\hline
\end{tabular}

O gráfico da Figura 3 mostra a relação entre o tamanho de partícula $\left(D_{90}\right)$ e o tempo de moagem. Pode-se verificar que a medida que o tempo de moagem aumenta o tamanho de partícula torna-se menor. Isso indica que o mecanismo de moagem sobrepõe o efeito de soldagem observado em moagem de alta energia, devido ao processo ocorrer em via úmida que diminui o calor gerado pelo processo (Suryanarayana, 2001).

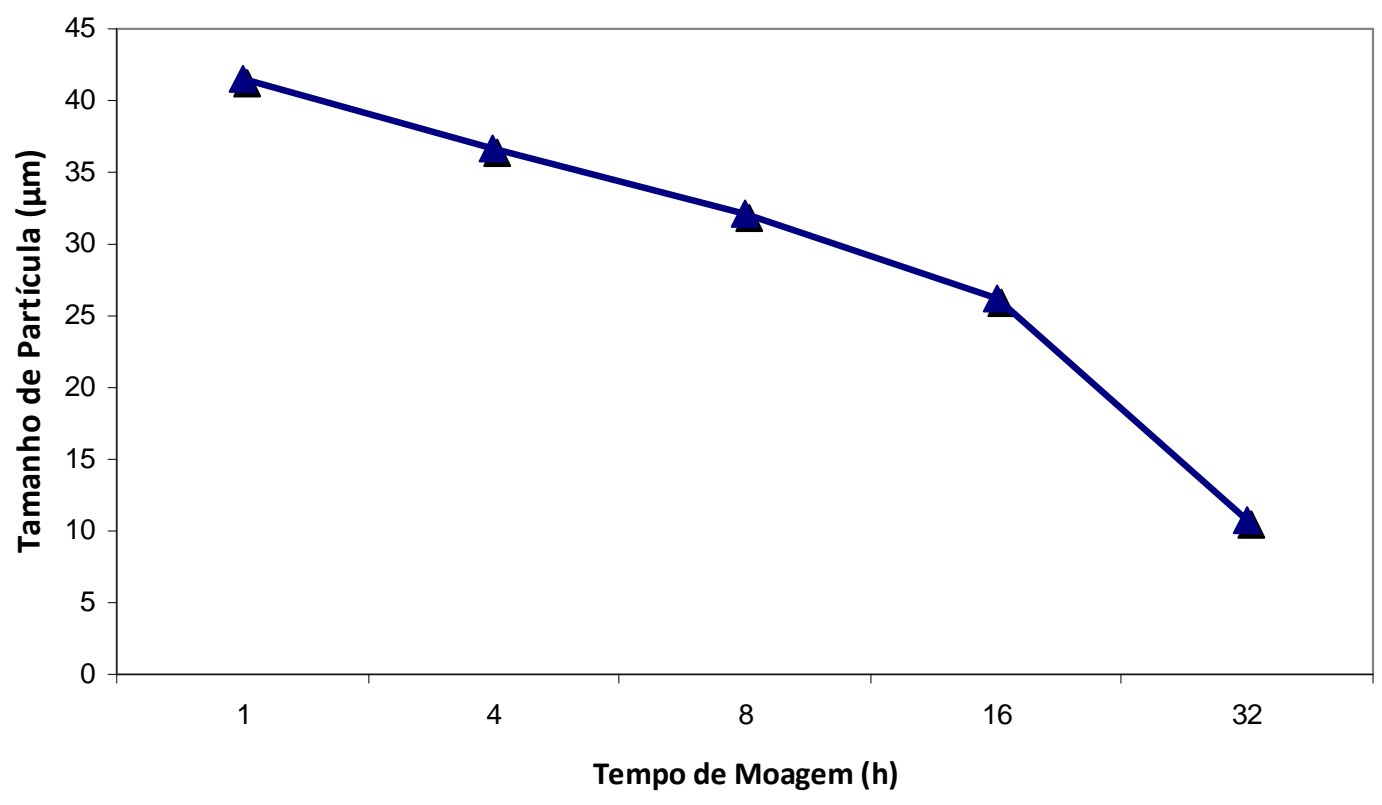

Figura 3: Gráfico do tamanho de partícula em função do tempo de moagem. 


\subsection{Evolução morfológica das partículas de pó do compósito}

A técnica escolhida para obtenção do compósito proposto Mo-30\%Cu permite avaliar a evolução da morfologia das partículas com o tempo de moagem. A evolução morfológica pode ser observada na Figura 4 (a)-(e).
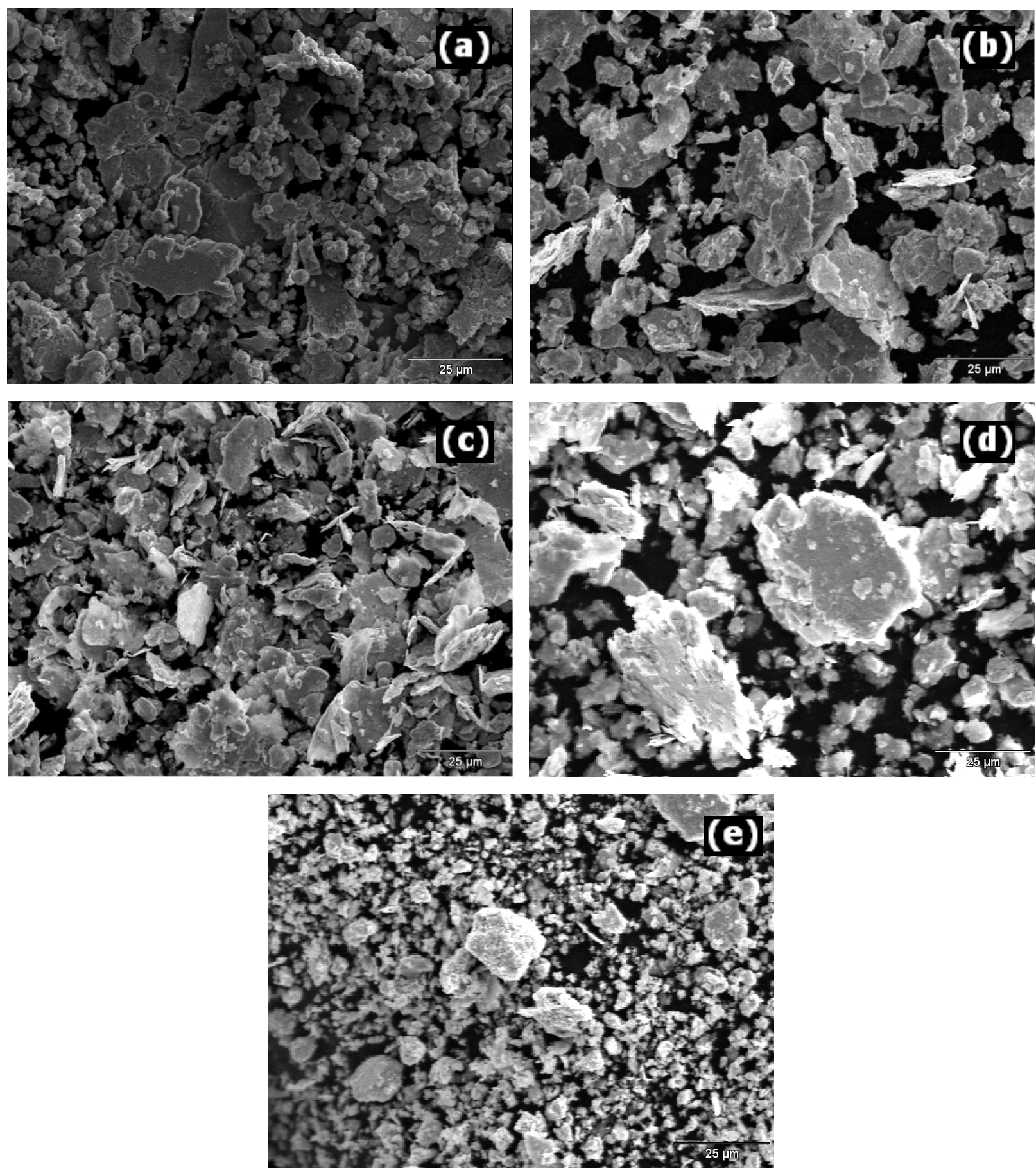

Figura 4: Micrografia do pó de Mo-30\%Cu, moído por: (a) 1 hora, (b) 4 horas, (c) 8 horas, (d) 16 horas e (e) 32 horas.

Nestas micrografias, observa-se inicialmente a formação de aglomerados com tamanhos que variam entre 5 a $20 \mu \mathrm{m}$ misturados com partículas menores do que $5 \mu \mathrm{m}$. Os tamanhos, tanto das partículas 
como dos aglomerados, são menores do que os tamanhos das partículas dos elementos utilizados para obter os compósitos produzidos.

Comparando as micrografias nas Figuras 4 (a)-(e) verifica-se uma redução do tamanho de partículas agregadas com o decorrer da moagem. Isto mostra que é possível controlar a redução do tamanho de partícula através do tempo de moagem.

\subsection{Ensaio de compressibilidade}

A Figura 5 mostra o efeito da pressão de compactação na densidade relativa das amostras em temperatura ambiente. Todas as amostras apresentaram aumento da densidade relativa com o aumento da pressão, com o tempo de moagem de 1 hora mostrando maiores valores de densidade para cada pressão aplicada.

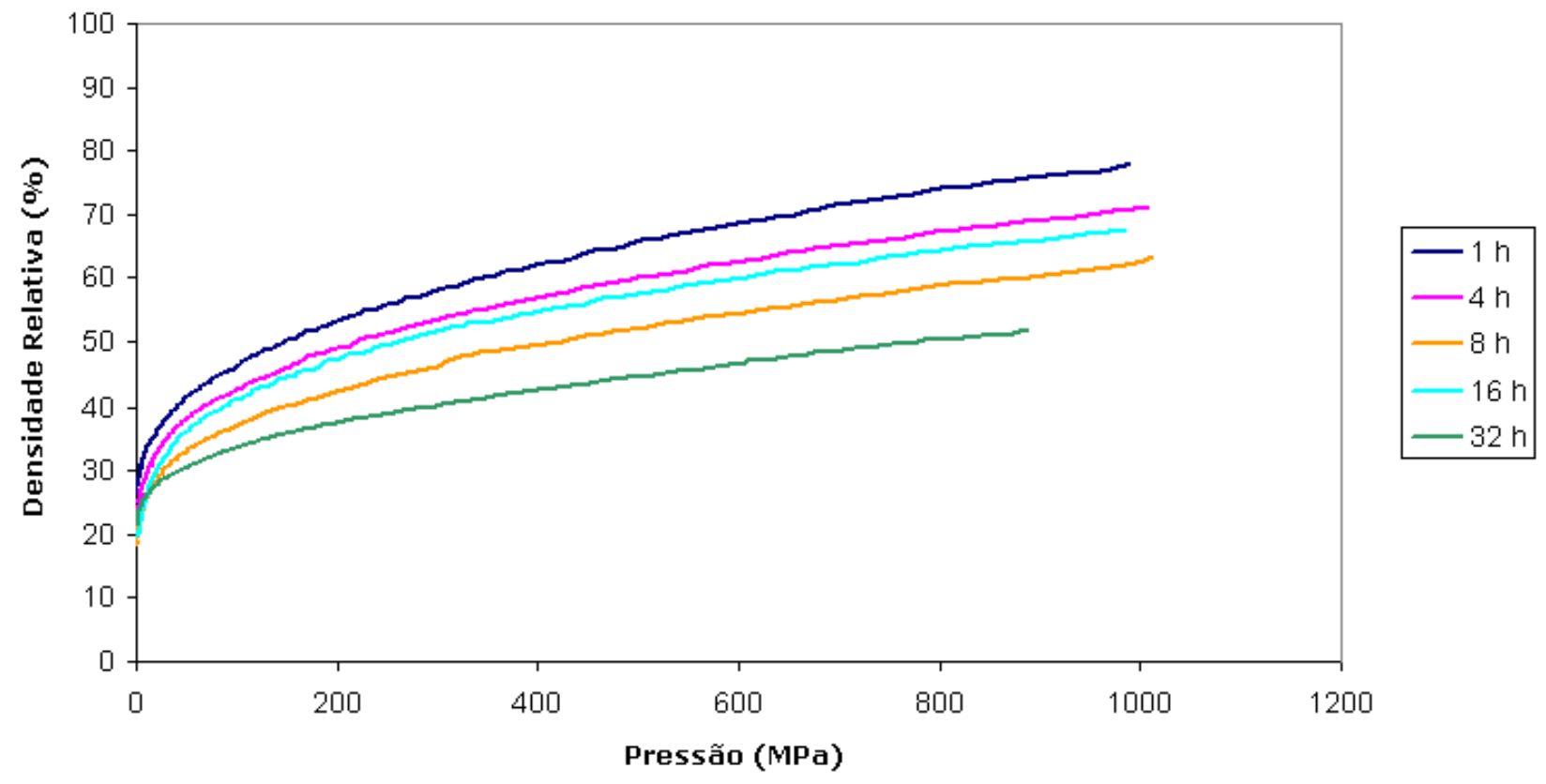

Figura 5: Curva de compressibilidade das misturas de Mo-30Cu.

A morfologia das partículas influencia a compressibilidade do pó. Partículas com morfologia achatada melhoram a capacidade de deformação durante a compactação (Hewitt et al., 2009). Assim, entre todos os tempos de moagem realizados neste estudo, a maior densidade relativa é alcançada pelo pó moído por 1 hora, resultado de sua morfologia achatada, que reflete numa área específica maior e que consequentemente aumentou a união mecânica entre as partículas. O encruamento sofrido pelas partículas durante o processamento por moagem também é uma das causas na queda da compressibilidade do material. 


\section{Conclusões}

Com o aumento do tempo de moagem ocorreu a diminuição do tamanho de partícula seguida de formação de aglomerados com tamanhos menores do que os pós elementares utilizados na obtenção dos compósitos. Além disso, os resultados mostraram que o controle do tamanho de grão do pó compósito pode ser feito através do tempo de moagem.

As imagens de MEV permitiram visualizar os efeitos do processamento de moagem de alta energia, como a alteração de morfologia das partículas e o seu refinamento, além da formação de aglomerados de partículas finas.

Todas as misturas apresentaram a curva típica de compressibilidade, na qual a densidade aumenta com o aumento da pressão de compactação, enquanto que a inclinação da curva diminui com o aumento da pressão. Um menor tamanho de partícula causa uma diminuição da compressibilidade para o compósito estudado, assim como o fato do material aumentar seu encruamento com o tempo de moagem.

As características apresentadas pelas misturas obtidas demonstram a necessidade de um tratamento térmico para que ocorra a recuperação e recristalização do material encruado. Esta ainda deve ser feita em atmosfera de hidrogênio de forma a remover qualquer oxidação que possa ter surgido durante este processamento.

\section{Agradecimentos}

Os autores agradecem aos órgãos financiadores CNPq e CAPES.

\section{Referências}

CAI, S.; MA, X.; TANG, H.; ZHAO, W.; YAN, J.; ZHAO, B.; QIAO, Z. 2007. Preparation of W-Al-Mo Ternary Alloys by Mechanical Alloying. Journal of Alloys and Compounds, 430:77-80.

CHEN, W.; KAN, Z.; DING, B. 2005. Preparation and Arc Breakdown Behavior of Nanocrystalline W-Cu Electrical Contact Materials. Journal of Materials Science \& Technology, 21:6.

HEWITT, S.A.; LAOUI, T.; KIBBLE, K.K. 2009. Effect of Milling Temperature on the Synthesis and Consolidation of Nanocomposite WC-10Co Powders. International. Journal of Refractory Metals and Hard Materials, 27:66-73.

MARTÍNEZ, V.P.; AGUILAR, C.; MARIN, J.; ORDOÑEZ, S.; CASTRO, F. 2007. Mechanical Alloying of Cu-Mo Powder Mixtures and Thermodynamic Study of Solubility. Materials Letters, 61:929-933.

ROTTA, M. 2005. Compósito Nb-Cu Obtido por Ação Mecânica e Sinterização - Comportamento Físico, Térmico e Elétrico. Maringá, PR. Dissertação de mestrado. Universidade Estadual de Maringá, 90 p. 
SCHUBERT, T.; TRINDADE, B.; WEIBG“ARBER, T.; KIEBACK, B. 2008. Interfacial Design of Cu-Based Composites Prepared by Powder Metallurgy for Heat Sink Applications. Materials Science and Engineering A, 475:39-44.

SONG, P.; CHENG, J.; WAN, L.; ZHAO, J.; WANG, Y.; CAI, Y. 2008. Preparation and Characterization of Mo$15 \mathrm{Cu}$ Superfine Powders by a Gelatification-Reduction Process. Journal of Alloys and Compounds, 476:226-230.

SURYANARAYANA, C. 2001. Mechanical Alloying and Milling. Progress in Materials Science, 46:1-184. 